\title{
Exploring Brain-to-Brain interaction at a distance: a global or differential relationship?
}

V.4

William Giroldini ${ }^{1,2}$, Luciano Pederzoli ${ }^{1,2}$, Marco Bilucaglia ${ }^{1}$, Elena Prati ${ }^{1}$ and Patrizio Tressoldi ${ }^{2}$

${ }^{1}$ EvanLab, Firenze, Italy

${ }^{2}$ Science of Consciousness Research Group, Dipartimento di Psicologia Generale, Università di Padova, Italy

Corresponding author:

Patrizio Tressoldi

patrizio.tressoldi@unipd.it 


\begin{abstract}
Backgound: The main objective of this exploratory study was a confirmation of the results obtained by Giroldini et al, 2016, relative to the possibility of identifying a long-distance connection between the EEG activities of two totally isolated subjects, one of whom was stimulated with light and sounds.
\end{abstract}

In this new study we have used the method of the steady-state stimulus (visual and auditory) given at the frequencies of 10,12 and $14 \mathrm{~Hz}$ in order to answer the following questions:

- What is the relationship between the power of the EEG response in the stimulated partner and that of the other isolated partner?

- Is the relationship between the EEG activities of the stimulated and the isolated partner global (i.e., an undifferentiated response), or is it differentiated and thus displays variations depending on the characteristics of the stimulation applied to the stimulated partner?

Methods: Five adults chosen for their experience in mind control techniques and their mutual friendships took part in this study. Each participant took turns in being both the stimulated partner and the isolated non-stimulated partner with each of the others, making a total of 20 pair combinations.

The stimulated partner received three blocks of 32 visual-auditory stimulations lasting 1 second modulated at $10 \mathrm{~Hz}, 12 \mathrm{~Hz}$, and $14 \mathrm{~Hz}$ respectively, with a constant inter-stimulus interval of 4 seconds.

The EEG activity of each pair was recorded at 128 samples/sec over 14 channels and analyzed by measuring traditional steady-state potentials and the Pearson's linear correlation between all possible signal pairs with an innovative algorithm.

Results: From the results of twenty pairs of subjects, we found an overall increase in the correlation among the EEG channels of the isolated partners, therefore confirming the previous research. Furthermore, we did not find any correlation between the correlation strength among the EEG channels of the stimulated partner and that observed in the non-stimulated partner, suggesting that this physical characteristic cannot be transferred between isolated partners; but we did find that the correlation among the EEG channels of the isolated distant partners changed not only globally, but also when the frequencies perceived by the stimulated partners were outside the Alpha band, suggesting that this neurophysiological mental connection at a distance may be differentiated.

Keywords: EEG, mind-to-mind interaction at a distance, Generalized Quantum Theory, Steadystate potentials. 


\section{Introduction}

The hypothesis that the human mind exhibits "quantum-like" characteristics - that is, similar to those shown experimentally in quantum physics with photons, electrons, or atoms, and more recently in biology - is clearly hazardous, but experimentally verifiable. The aforementioned characteristics appear to violate the laws of classical physics, as in the entanglement phenomenon, in which the components of a system show a special relationship despite being physically separated in space or even time, without the possibility of a connection by classical means such as electromagnetic waves (Horodecki, Horodecki, Horodecki, \& Horodecki, 2009; Lambert et al., 2012).

For example, the possibility that the brain activities of two physically distant, but emotionally and mentally connected individuals, can display a correlation in the absence of any normal sensory connection has been supported by Giroldini et al., (2016) and independently confirmed by Radin, (2017) and a further approximately thirty studies (see Table S1 in the study of Giroldini et al., 2016a).

In a typical study of this kind, two members of a pair are separated (varying from meters to kilometers) and sensorially isolated from each other. One member is stimulated with either structured (e.g. images) or unstructured (e.g. lights and sounds) information, and the correlation between their respective EEG activities is measured. For example, if the sensorially isolated partner's EEG shows a variation correlated to the stimulated partner's EEG, we can assume (unless potential artefacts are discovered) that it is proof of a non-local (long-distance) connection between the two brains.

Even if, from a phenomenological point of view, this correlation seems to show a causal effect of the stimulated partner upon the sensorially isolated one, some authors believe it to be a form of biological entanglement similar to what occurs in quantum physics (see for example Walach, Tressoldi, \& Pederzoli, 2016), and therefore is an expression of an acausal correlation.

However, to date, the relationship between the characteristics of the stimulated partners' signals and the same parameters seen in the isolated non-stimulated distant partners have yet to be examined in depth, except for the fact that the latter signal is much weaker (by approximately a factor of ten). This study is an exploratory contribution to better understand this relationship. We specifically sought to answer the following questions:

-What is the relationship between the intensity (or power) of the observed EEG signal between the stimulated partner and the distant isolated partner?

-Is the relationship between the EEG activities of the stimulated and distant isolated partners global (i.e., an undifferentiated response), or is it differentiated and therefore changes 
depending on the characteristics of the stimuli applied to one of the pair?

The answers to these two questions are important for a better understanding of which characteristics of the stimulation can be detected in the two separate brains.

\section{Materials and Methods}

\section{Participants}

Five adults - two women and three men - took part in this study, with an average age of 38.3 years $(\mathrm{SD}=7.5)$, chosen for their experience in mind control techniques (mainly meditation) and their mutual friendship. We consider these pre-requisites essential for an adequate "mental and emotional connection" between the pairs. Each participant took turns in being both the stimulated partner and the non-stimulated partner with each of the others, making a total of 20 pair combinations. This sample size of 20 pairs of participants was estimated by setting the following parameters: statistical power $=.80$, a one-tailed Type I error $=.05$ and an expected effect size $d=.5$ for a one sample t-test (difference from a constant $=0$ : http://powerandsamplesize.com/Calculators/Test-1 Mean/1-Sample-1-Sided).

\section{Statement of Ethics}

The use of experimental subjects is in accordance with ethical guidelines as outlined in the Declaration of Helsinki, and the study has been approved by the Ethical Committee of the University of Padova's Department of General Psychology, prot. n. 63, 2012. Before taking part in the experiment, each subject gave his/her informed consent in writing after having read a description of the experiment.

\section{EEG equipment}

Two Emotiv Epoc ${ }^{\mathrm{TM}}$ EEG devices were used, modified to allow connection (via multi-contact connectors) to professional Bionen headsets (See Figure S1 in the Supplemental Information) to ensure high quality EEG signals. The system's accuracy and signal quality were thoroughly checked and ascertained. The sample frequency was 128 samples/sec over 14 channels connected to locations Fp1, F3, C3, P3, O1, F7, T5, Fp2, F4, C4, P4, O2, F8, T6. The instruments were provided with a built-in fifth order low-pass digital filter (bandwidth from 0.2 to $45 \mathrm{~Hz}$ ), as well as two notch filters at 50 and $60 \mathrm{~Hz}$ respectively as protection against noise produced by the local electricity network.

Signal acquisition by the two EEG devices was controlled by a specially designed software program with an acquisition synchronicity precision better than $1 / 128$ second. The Emotiv ${ }^{\circledR}$ EEG has a proprietary wireless network connection at a frequency of $2.4 \mathrm{G} \mathrm{Hz}$, thus ensuring total electrical 
independence and separation between the two devices.

The experiment was conducted at the EvanLab laboratory in Florence (Italy), which is comprised of two separate sound- and lightproof rooms with no electromagnetic disturbances (see Figure 1).

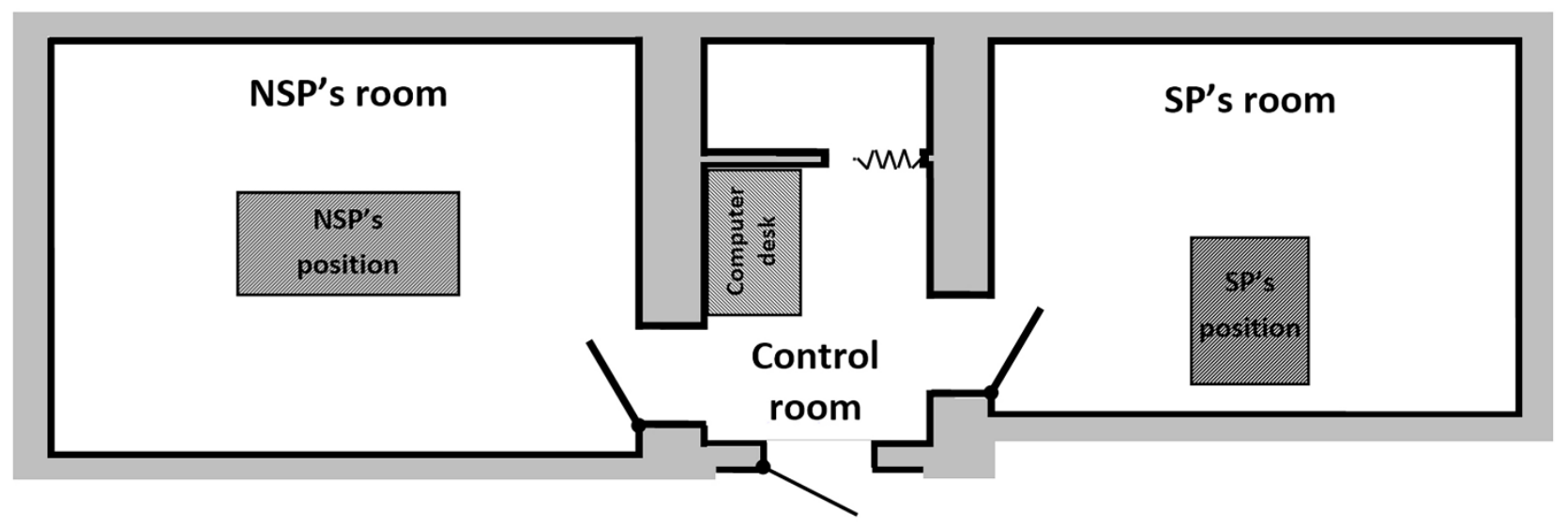

Figure 1: floor plan of EvanLab laboratory. $\mathrm{SP}=$ stimulated partner; NSP = non-stimulated partner

\section{Visual-auditory stimulation}

The visual-auditory stimulations were conducted in three blocks of 32 stimulations lasting 1 second, at the same time on-off modulated at $10 \mathrm{~Hz}, 12 \mathrm{~Hz}$, and $14 \mathrm{~Hz}$ respectively, with a duty cycle of $50 \%$ for both sound and light, and with a constant inter-stimulus interval of 4 seconds.

The audio modulation was performed on a $900 \mathrm{~Hz}$ sinusoidal carrier and applied through $32 \mathrm{ohm}$ impedance earphones at a volume of about $80 \mathrm{~dB}$.

Several tests showed that no sound could be perceived by a person even at a distance of half a meter from the stimulated subject. Consequently, taking also into account the thick walls and the two sound-proof doors, no significant stimulus sound could be transferred between the two subjects. The method of stimulus administration based on a modulation frequency from 4 to $20 \mathrm{~Hz}$ is also called "Steady-State" (Pastor, Artieda, Arbizu, Valencia, \& Masdeu, 2003; Picton, John, Dimitrijevic, \& Purcell, 2003).

The interval between the three blocks was randomly varied at between 40 and 90 seconds. The visual stimulus was provided by an array of 16 red LEDs positioned about $30 \mathrm{~cm}$ from the stimulated partner's closed eyes, while the sound was simultaneously sent directly to the ears via the above-mentioned $32 \mathrm{ohm}$ impedance earphones. The three frequency blocks were given randomly without repetition of the same frequency.

The raw data are available at: http://tiny.cc/owzyly

\section{Procedure}

The stimulated partner was given the following instructions: "When you are ready, relax with your 
eyes closed and be prepared to receive a visual and auditory stimulus connecting mentally and with positive emotions with your partner. Limit your body movements to prevent interference with your EEG activity. You will perceive three blocks of 32 stimulations of 1 second each, the blocks will be separated by long random pauses in order to avoid predictable rhythms. The experiment will last about 15 minutes."

The non-stimulated partner was given the following instructions: "When you are ready, relax with your eyes closed and connect mentally with positive emotions with your partner, who is receiving visual and auditory stimulations. Keep your body still to prevent interference with your EEG activity. The experiment will last about 15 minutes."

At the end of each trial involving pairs, their roles were reversed.

\section{Timing of the stimuli}

Tests performed later on the data acquisition process showed a slight shift of the presentation of the stimulus with respect to the theoretical instant of stimulation. This shift is caused by the software program's execution features due to the operating system (Windows 10).

The shift is equal to around 10 samples ( $\sim 0.08$ secs $)$ and can easily be compensated for during data analysis.

The images on the left in Figure 2 are obtained by measuring the signals from a photodiode in front of the stimulus LEDs to obtain time references, which are then fed back into the Emotiv Epoc ${ }^{\mathrm{TM}}$. From these measurements, it is possible to completely compensate for the shift.
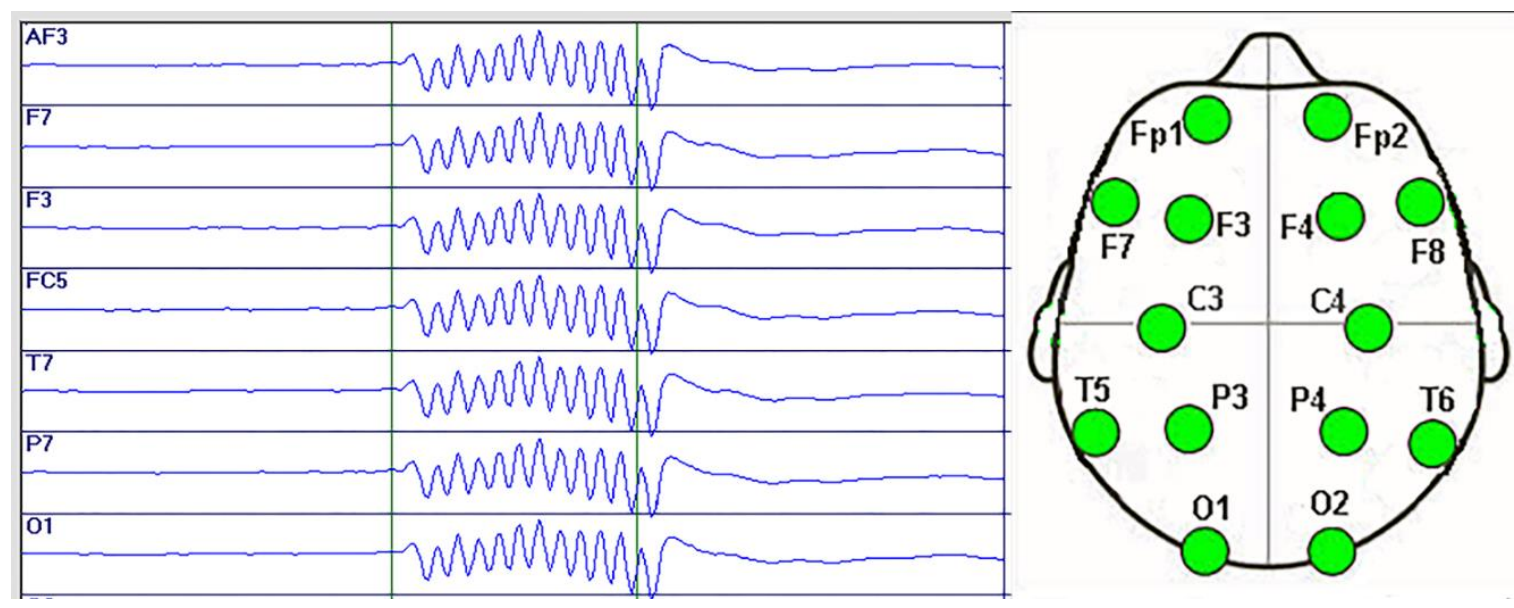

Figure 2: The signal obtained from a BPW34 photodiode in front of the illuminator lights shows a delay between the stimulus command and its actualization (about 10 samples, or 0.08 secs). It is taken into account by adding up the time at the start of the stimulation period. In the image, the stimulus was at $12 \mathrm{~Hz}$. On the right is a diagram showing placement of the EEG's 14 channels. 


\section{Stimulated partners EEG data analysis}

Since each recording contained 32 stimulations for each of the three different frequencies, each of the three was processed the same way. One of the first types of analysis used was the FFT (Fast Fourier Transform) applied to a 3 second period, comprised of a pre-stimulus 1 second period, followed by a 1 second stimulus, and then a 1 second post-stimulus period, finally averaged over all stimuli (32 for each frequency in each of 20 files).

Next the FFT differentials were calculated - that is, the differences in frequency composition between the stimulus period and the pre-stimulus period. The post-stimulus period was ignored. Generally, in directly stimulated subjects, the FFT shows peaks very close to the stimulus frequencies $(10,12$, and $14 \mathrm{~Hz})$ and their potential harmonics, although they are only $10-15 \%$ bigger than the baseline, see Figures 3, 4, and 5.

This means the stimulation effect is not strong enough to be seen without appropriate processing. So as to better highlight the effects, the differences between the two situations (stimulus and prestimulus) were calculated and, once amplified, the stimulation frequencies became clear. All three stimulation frequencies show a strong reduction in the subjects' spontaneous Alpha frequency (the well-known typical Alpha-block).

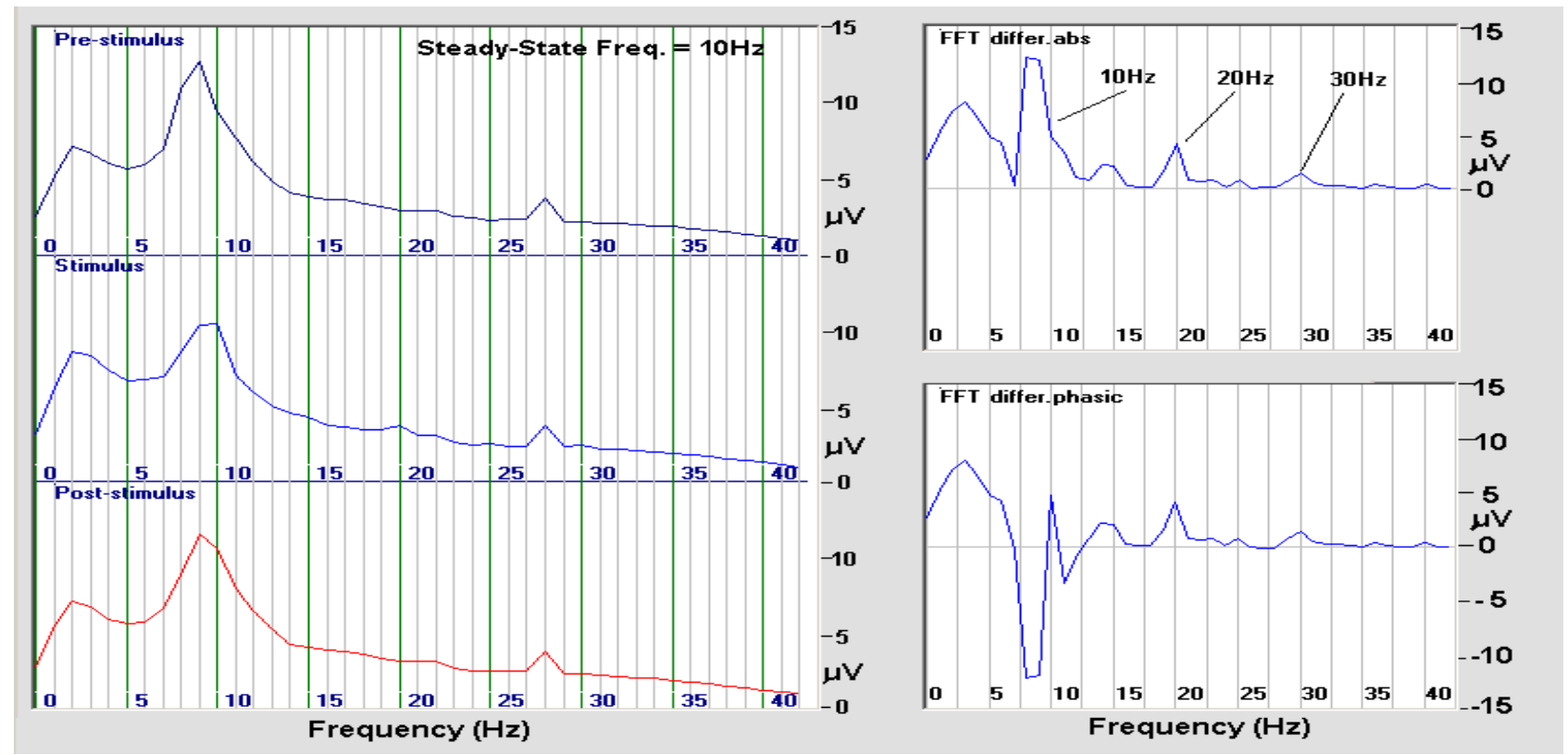

Figure 3: Stimulated partners at $10 \mathrm{~Hz}$ : on the left the FFT (between 1 and $42 \mathrm{~Hz}$ ) of the prestimulus, stimulus, and post-stimulus periods. On the right the differential graphs showing the loss of spontaneous Alpha and the presence of stimulus frequency including two of its harmonics, at 20 $\mathrm{Hz}$ and $30 \mathrm{~Hz}$. 

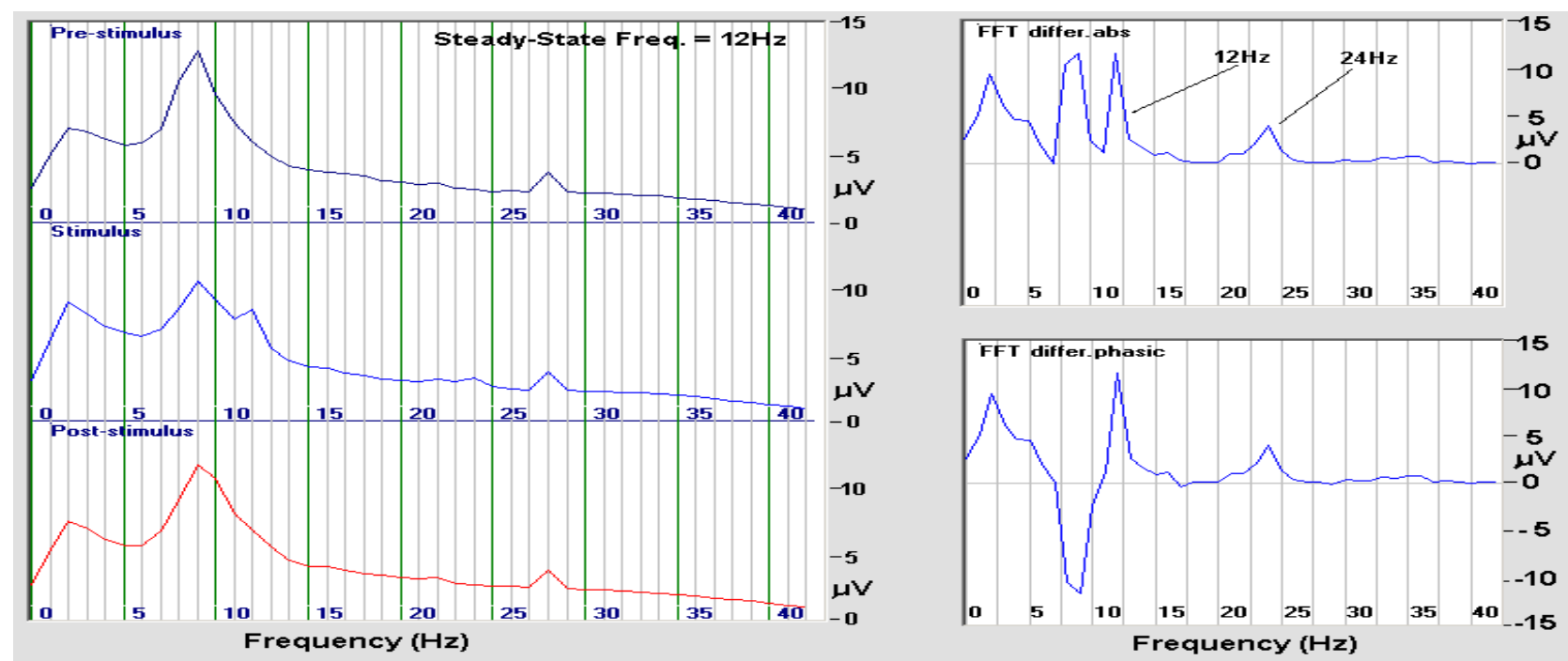

Figure 4: Stimulated partners at $12 \mathrm{~Hz}$ : on the left the FFT (between 1 and $42 \mathrm{~Hz}$ ) of the prestimulus, stimulus, and post-stimulus periods. On the right the differential graphs showing the loss of spontaneous Alpha and the presence of stimulus frequency including its first harmonic at $24 \mathrm{~Hz}$.
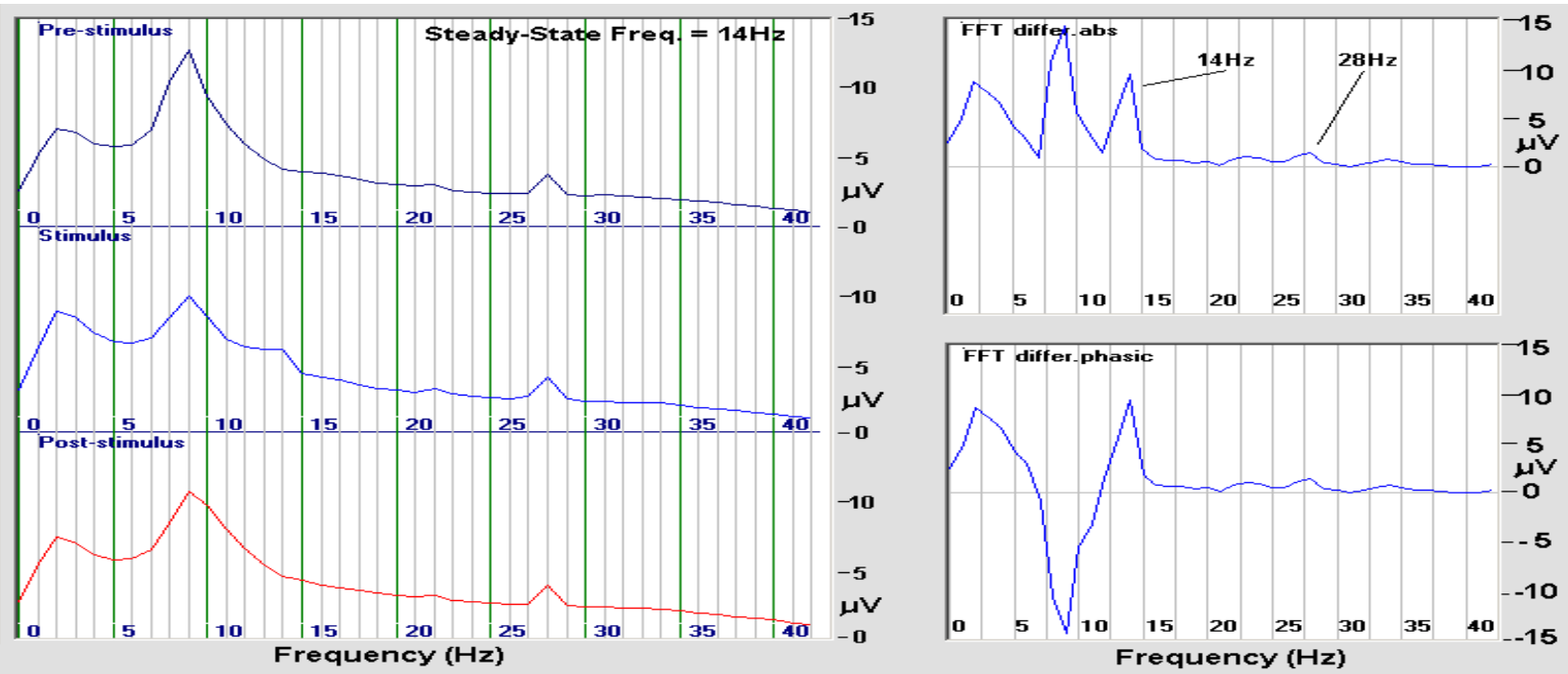

Figure 5: Stimulated partners at $14 \mathrm{~Hz}$ : on the left the FFT (between 1 and $42 \mathrm{~Hz}$ ) of the prestimulus, stimulus, and post-stimulus periods. On the right the differential graphs showing the loss of spontaneous Alpha and the presence of stimulus frequency including its first harmonic at $24 \mathrm{~Hz}$.

A small peak at $28 \mathrm{~Hz}$ appears only in stimulated partners, probably due to a weak disturbance at 50 $\mathrm{Hz}$ from the power source for the LED array used for visual stimuli (the difference between the 128 $\mathrm{Hz}$ sampling frequency and the second harmonic of $50 \mathrm{~Hz}$ ) or a second harmonic of $14 \mathrm{~Hz}$. The 28 $\mathrm{Hz}$ peak is however eliminated by the differential FFT.

\section{Analysis of stimulated partners EEG data using the GW6 method.}

All EEG signals were filtered (1 Hz width) at the stimulation frequency $(10 \mathrm{~Hz}, 12 \mathrm{~Hz}$ or $14 \mathrm{~Hz}-$ see Figure 6) by an eight-order band-pass Butterworth filter, in order to implement a time-reversal 
filter to ensure a zero phase delay. Therefore, the classic ERP was identified in the stimulated partners through simple averaging procedure, calculating the power, and finally extracting a multiple correlation value between EEG channels in accordance with the GW6 method.

To briefly outline here the fundamentals of this method, it is based on calculating Pearson's linear correlation between all channel pairs, from which pairs of fixed duration data segments of about $250 \mathrm{~ms}$ are extracted, examining 4 seconds of consecutive data $(1.5 \mathrm{~s}$ pre-stimulus, $1 \mathrm{~s}$ stimulus, 1.5 s post-stimulus).

These segment pairs (sliding windows) are then slid along the time axis of the two channels (for all the possible combinations), generating a series of curves $\mathrm{R}(\mathrm{I}, \mathrm{X})$, where I represents pair combinations, $\mathrm{I}=91$ in this case (14 electrodes $\mathrm{x}$ 13/2) and $\mathrm{X}$ is time. Subsequently this series of curves is processed to produce a single graph $\operatorname{Sync}(\mathrm{x})$, that basically represents the global variations of correlation (or synchronization) between all EEG channels, using suitable pre- and post-stimulus periods as a baseline. The details of the method GW6 are described in Giroldini et al., (2016).

The EEG signals were filtered in a narrow band (1 Hz width) centered at the stimulation frequency (i.e. $10 \mathrm{~Hz}, 12 \mathrm{~Hz}, 14 \mathrm{~Hz}$ - see Figure 6). The GW6 graph, the ERP's power and the signal power were calculated as the average of all stimuli and all subjects.

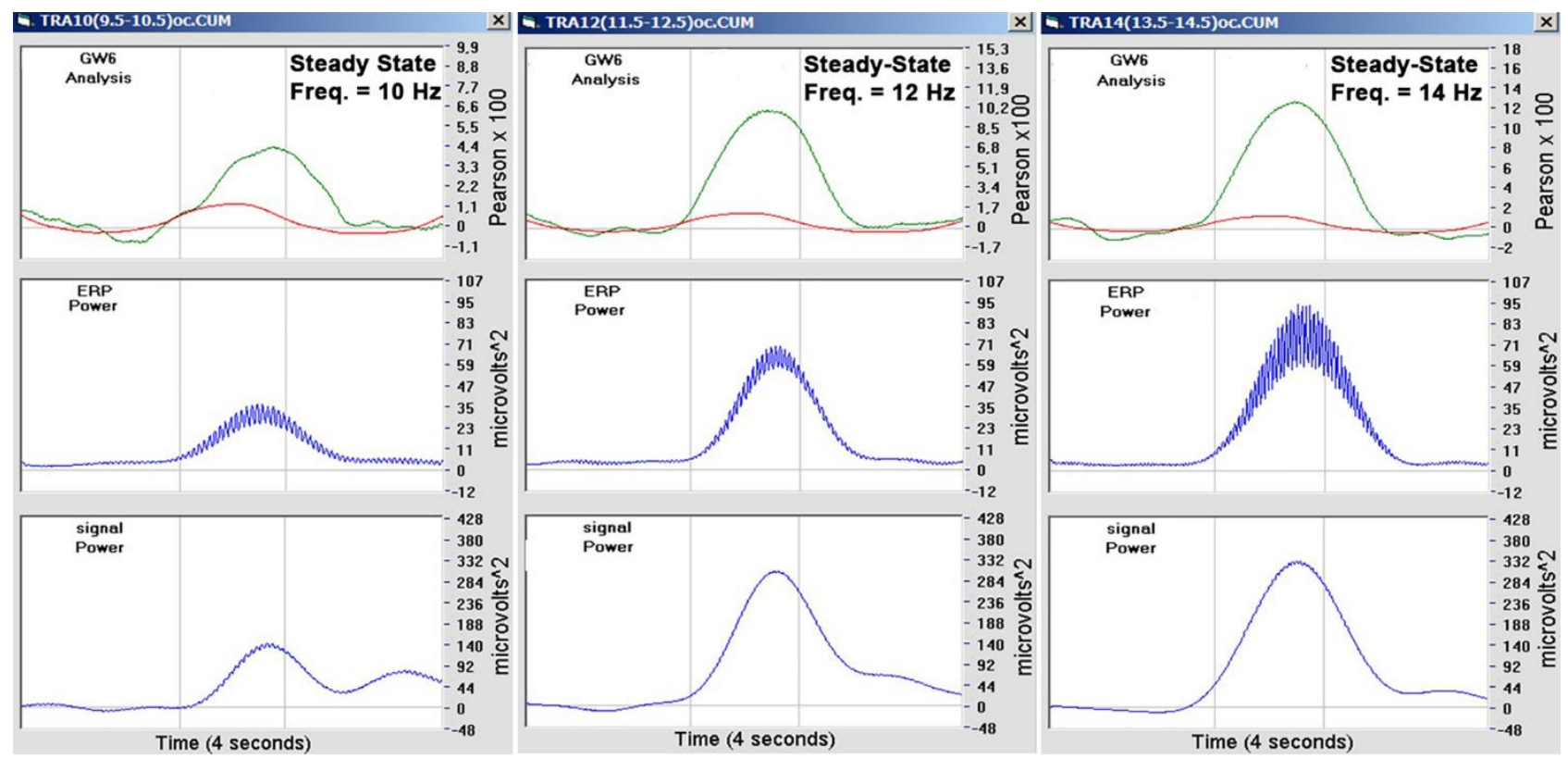

Figure 6: Stimulated partners: averaged ERP graphs of 32 stimuli over 20 files of stimulated partners, with the signal filtered respectively in bands $9.5-10.5 \mathrm{~Hz}$ (left) $11.5-12.5 \mathrm{~Hz}$ (middle) and $13.5-14.5 \mathrm{~Hz}$ (right), therefore centered at $10 \mathrm{~Hz}, 12 \mathrm{~Hz}$ and $14 \mathrm{~Hz}$ respectively. The red curves in the upper graphs represent the expected random chance.

Looking at the graphs shown in Figure 6, it is possible to clearly see the normal response to the stimuli, for example the power of classic ERP, or the signal power. It is also important to stress that the greatest response is obtained by effectively filtering the signal at the true stimulus frequency 
(e.g. 9.5-10.5 Hz for the steady-state frequency of $10 \mathrm{~Hz}$ ).

If we filter within a frequency range even slightly shifted (e.g. 10.0-11.0 Hz), the response is always reduced. In short, the greatest steady-state response exactly coincides with the stimulus frequency. From Figure 6, we note that the curve's height increases as it moves from 10 to $14 \mathrm{~Hz}$, probably because the stimulation frequency moves away from that of the spontaneous Alpha band frequencies $(\sim 8-12 \mathrm{~Hz})$ of the participants.

The continuous red line, together with the green curve generated by GW6, represent the random expectation (calculated using a method described later). In all cases, we see that the GW6 graph greatly exceeds the random expectation, or "zero curve".

\section{Analysis of non-stimulated partners EEG data.}

EEG analysis of the non-stimulated partners did not evidence any significant peaks at stimulation frequencies in differential FFT graphs equivalent to those presented in Figures 3, 4 and 5.

Furthermore, on average there are no peaks resembling a classic ERP, even when power is taken into account. In the EEG signal power there are only weak and variable fluctuations coinciding with the remote stimulus, and therefore there is no response which can be definitely similar or correlated to that obtained in stimulated partners.

The only graph showing some significant variation with respect to baseline EEG activity is that obtained using the GW6 method, by filtering $0.5 \mathrm{~Hz}$ below the stimulus frequency (see Figure 7). For example, in order to identify a more significant variation in the band around $10 \mathrm{~Hz}$, it was necessary to filter (using the same band-pass filter described for stimulated partners) in the range from 9.0 to $10.0 \mathrm{~Hz}$. The same applies to other stimulation frequencies. This frequency shift in the non-stimulated partner's response does not appear to be due to the software, because the same program, when applied to stimulated partners, shows the maximum response peak exactly at the stimulation frequency. 

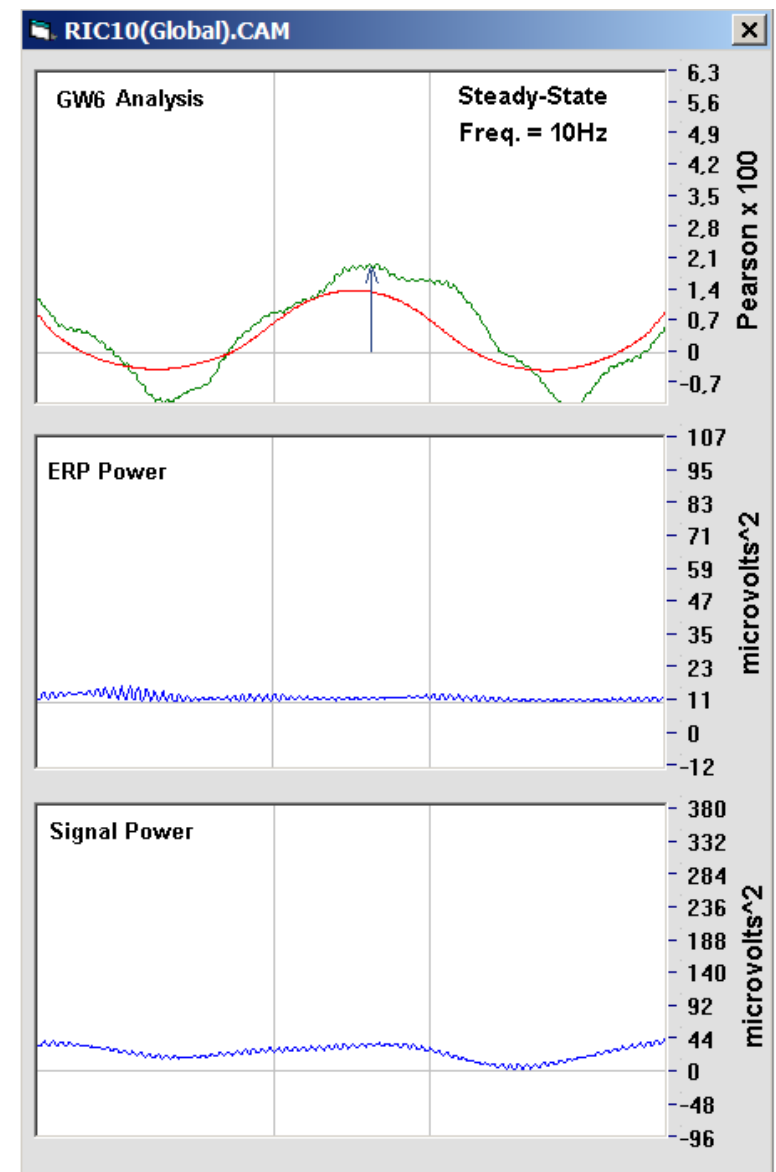

G. RIC14(global).CAM
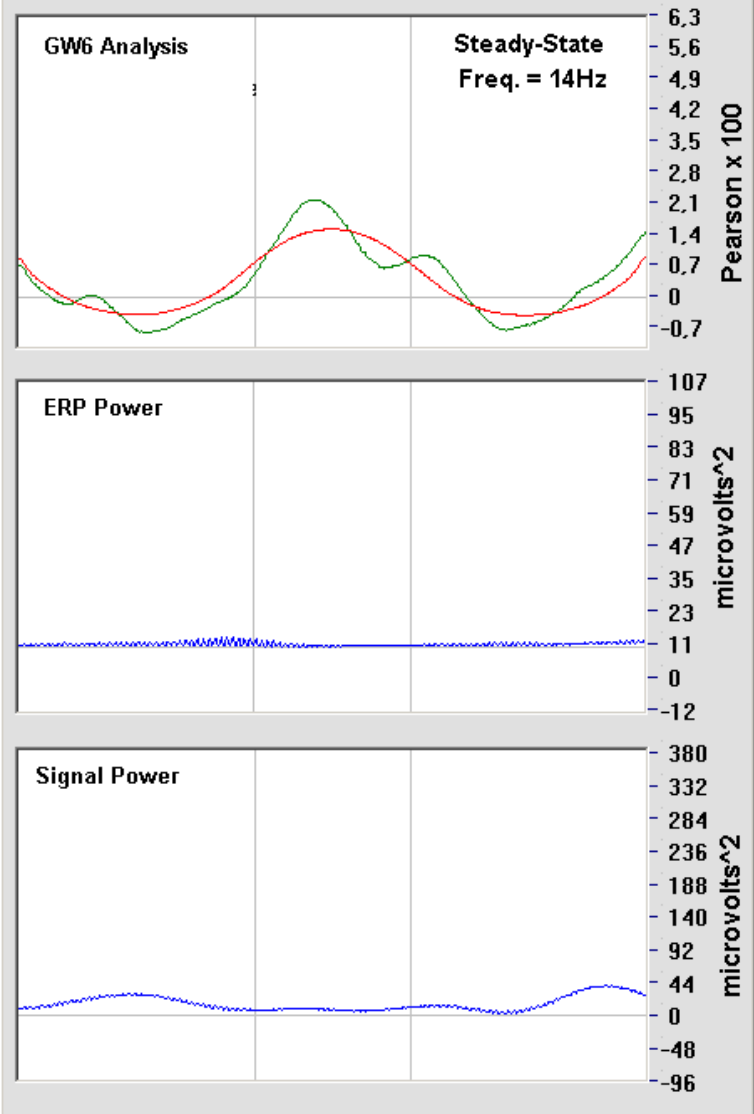
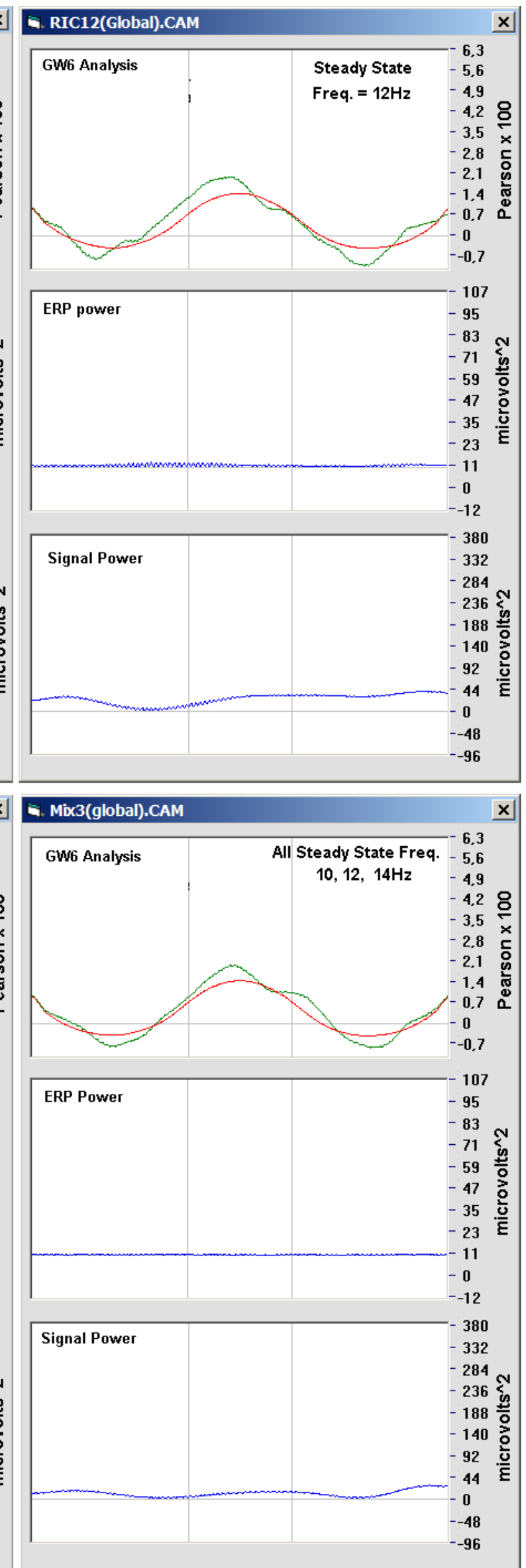

Figure 7: Non-Stimulated partners: averages of 32 stimuli for 20 files filtered in the 9-10 Hz band (top left); filtered in the 11-12 Hz band (top right); filtered in the 13-14 Hz band (bottom left) and average of the previous graphs (Overall Sync 1 curve: $20 \times 3=60$ files, bottom right). 
Note that the typical ERP curve and the ERP power curve are virtually flat.

Only the GW6 method reveals some significant deviations from what is expected from chance alone (red curve), mainly at $10 \mathrm{~Hz}$ and $14 \mathrm{~Hz}$, and in the global curve based on putting together the responses of all frequencies.

By applying the GW6 algorithm to each subject for each remote stimulus condition, 3 global curves called Sync1 were produced. Then the average of all the curves (based on 20 x $3=60$ Sync1 curves) was calculated, producing an overall Sync1 curve. From these resulting 4 curves, the maximum correlation value and its standard deviation for each frequency was calculated (see the arrow in top left of figure 7).

In order to compare these observed experimental values with a random estimate, for each subject and each stimulus condition a new dataset of 180 Sync1 curves was created based on randomly positioning the stimulus zone within the EEG recording, and using the same number of real stimuli (32 stimuli for each stimulus condition), obtaining a so-called "random dataset" ( 20 x $180=3600$ random Sync1 curves for each frequency), then calculating the corresponding averaged "max correlation" and the standard deviation values. These "random" curves are displayed in Figure 7 as red curves.

Finally, using both a fully independent method (the Montecarlo procedure) and a paired t-test, we calculated the one-tailed probability that the value of the random maximum correlation would be greater than the experimental one. With the Montecarlo procedure, from the total of the Sync1 random curves (for each frequency) we randomly extracted 20 curves, averaged them, and from this calculated the maximum correlation value. If this value was higher than the experimental one, a counter was incremented. This routine was repeated 2000 times.

The ratio between the final counter value and 2000 (the number of repetitions) is a direct representation of the probability. The results are displayed in Table 1.

Table 1: comparison between experimental and random values.

\begin{tabular}{cccccc}
\hline $\begin{array}{c}\text { Stimulus } \\
\text { Frequency }\end{array}$ & $\begin{array}{c}\text { Experimental } \\
\text { max correlation } \\
\text { Mean (SD) }\end{array}$ & $\begin{array}{c}\text { Random } \\
\text { max } \\
\text { correlation } \\
\text { Mean (SD) }\end{array}$ & $\begin{array}{c}\text { paired } \\
\text { t-test }\end{array}$ & $\begin{array}{c}\boldsymbol{p} \text { value } \\
\text { (one- } \\
\text { tailed) }\end{array}$ & $\begin{array}{c}\boldsymbol{p} \text { value (one-tailed) } \\
\text { following the Montecarlo } \\
\text { procedure }\end{array}$ \\
\hline $\mathbf{1 0 ~ H z}$ & $1.95(2.32)$ & $1.38(2.04)$ & 1.24 & 0.16 & .24 \\
$\mathbf{1 2 ~ H z}$ & $1.96(1.86)$ & $1.40(1.93)$ & 1.30 & 0.1 & .20 \\
$\mathbf{1 4 ~ H z}$ & $2.14(2.74)$ & $1.49(1.96)$ & 1.49 & 0.07 & .14 \\
$\mathbf{M i x 3}$ & $1.93(2.35)$ & $1.42(1.98)$ & 2.03 & 0.05 & .06 \\
\hline
\end{tabular}




\section{Correlation between the max correlation of the Stimulated vs that of the Non-stimulated partners}

As indicated in the introduction, the second aim of this study was to investigate the relationship between the strengths of the EEG Steady-state response between the stimulated partner and the distant isolated partner.

This relationship was analyzed by simple binary correlations between the values of the maximum correlations obtained with the GW6 algorithm of the stimulated and non-stimulated pairs. The values of Kendall's tau-b correlations are presented in Table 2.

Table 2: Values of the Kendall's tau-b correlations with corresponding credible intervals between the values of the maximum correlation of the stimulated (SP) and non-stimulated partners (NSP), for each of the three frequencies.

\begin{tabular}{l|c|c|c}
\hline & SPs 10 Hz & SPs 12 Hz & SPs 14 Hz \\
\hline NSPs $10 ~ H z$ & $-.33(-.56,-.006)$ & & \\
\hline NSPs $12 ~ H z$ & & $.09(-.2, .36)$ & \\
\hline NSPs $14 \mathrm{~Hz}$ & & & $-.04(-.32, .24)$ \\
\hline
\end{tabular}

Is it evident that the values of the correlations are very low, ranging from a minimum of -0.04 to a maximum of -.33, indicating an almost null correlation between the strength of the signals of the pairs of participants.

\section{Discussion}

The primary objective of this study was to confirm that the brain activities of two physically distant but emotionally and mentally connected individuals display a correlation in the absence of any normal sensory connection, as well as to obtain more information on the characteristics of their EEG signals.

Specifically, as mentioned in the introduction, we aimed at acquiring more information on the relationship between the observed signal strength in the EEG activities of the stimulated and nonstimulated partners as well as the possibility that this relationship between each partner's EEG activity is either undifferentiated, or differentiated, thus varying depending on the type of stimulation applied to the stimulated partners, in particular the frequency of the stimulus. This new experiment, conducted with the Steady-State method, confirms the results of Giroldini et al. (2016a) in which a sinusoidal sound frequency of $500 \mathrm{~Hz}$ and the same red LED array were used for stimulation, but neither of them was modulated in frequency. 
In these experiments, although the interstimulus time was constant ( 4 seconds), the time between the three blocks was random as was the order of the three frequencies. In these conditions we do not know of any mechanism able to generate a spurious correlation between the stimulus and (for example) the Alpha activity of the non-stimulated partner.

Regarding the relationship between the strength (Power spectrum, ERPs or correlations among the electrodes) of the EEG activities of the stimulated and non-stimulated partners, our results suggest that there is no such relationship and hence that this parameter cannot be communicated at a distance.

On the other hand, when taking into consideration the maximum correlation values as estimated by applying the GW6 algorithm, our results confirm the relationship between EEG signal characteristics of both the stimulated and non-stimulated partners, at least regarding the frequencies outside the Alpha band.

Indeed, in some preliminary tests we stimulated a partner with two frequencies, 15 and $18 \mathrm{~Hz}$, repeated 100 times. From the analysis of the non-stimulated partners, we found statistical significant results for both (see Supplemental Information).

At this stage of our studies we are unable to accurately determine which areas of the brain are the most sensitive to remote stimuli, even though we believe this may be due to the limitations of our mathematical analysis tools in distinguishing the actual signal from a strong background noise. We emphasize that this is an exploratory study and results were obtained after a series of unplanned post hoc choices, such as the extension of the filtration band $(1 \mathrm{~Hz}$ rather than $1.2 \mathrm{or} .8 \mathrm{~Hz})$, the size of the GW6 method's sliding window, the choice of individual vs overall values, etc.

\section{Conclusions}

Nonetheless, despite its limits, the information that emerges from this study can, if confirmed, provide important details about the relationship between the EEG activities of two physically distant and mentally connected partners.

To summarize, we can tentatively affirm that the EEG activity in non-stimulated partners generates a weak response with a signal to noise ratio that changes little as a function of the intensity of the response generated in the stimulated partners.

We can also affirm that the non-stimulated partner's response seems to be specific to the frequency induced in the EEG activity of stimulated partners.

The GW6 method, as well as similar ones based on the correlation of the EEG signals (e.g. Radin, 2017), seems suitable for this purpose, but we believe more refined tools are necessary, for example those based on machine learning algorithms (Müller et al., 2008; Stober, Sternin, Owen, \& Grahn, 2016), especially if we wish to detect signals after only a single stimulation and not, as we have 
done until now, after multiple repetitions of the same stimulation.

From a theoretical point of view, it seems fair to say that a mental connection at a distance can allow the transmission of some informational, rather than physical, characteristics of the shared signals. This seems plausible, given that we cannot theorize about any information transmission based on conventional electromagnetic waves. This interpretation fits well with a quantum-like mental and biological entanglement as predicted by the Generalized Quantum Theory (Walach, Tressoldi, \& Pederzoli, 2016) which predicts that quantum-like phenomena also take place in areas outside quantum physics - such as biology and psychology - based on exchange of information rather than of physical energy (Khrennikov, 1999). 


\section{References}

Giroldini, W., Pederzoli, L., Bilucaglia, M., Caini, P., Ferrini, A., Melloni, S., ... Tressoldi, P. (2016). EEG correlates of social interaction at distance. F1000Research, 4, 457. https://doi.org/10.12688/f1000research.6755.5

Giroldini, W., Pederzoli, L., Bilucaglia, M., Melloni, S., \& Tressoldi, P. (2016). A new method to detect event-related potentials based on Pearson's correlation. EURASIP Journal on Bioinformatics and Systems Biology, 11. https://doi.org/10.1186/s13637-016-0043-z

Horodecki, R., Horodecki, P., Horodecki, M., \& Horodecki, K. (2009). Quantum entanglement. Reviews of Modern Physics, 81(2), 865-942. https://doi.org/10.1103/RevModPhys.81.865

Jasp Team. (2017). JASP (Version 0.8.2.0)[Computer software].

Khrennikov, A. Y. (1999). Classical and Quantum Mechanics on Information Spaces with Applications to Cognitive, Psychological, Social, and Anomalous Phenomena. Foundations of Physics, 29(7), 1065-1098.

Lambert, N., Chen, Y.-N., Cheng, Y.-C., Li, C.-M., Chen, G.-Y., \& Nori, F. (2012). Quantum biology. Nature Physics. https://doi.org/10.1038/nphys2474

Müller, K.-R., Tangermann, M., Dornhege, G., Krauledat, M., Curio, G., \& Blankertz, B. (2008). Machine learning for real-time single-trial EEG-analysis: From brain-computer interfacing to mental state monitoring. Journal of Neuroscience Methods, 167(1), 82-90. https://doi.org/10.1016/j.jneumeth.2007.09.022

Pastor, M. A., Artieda, J., Arbizu, J., Valencia, M., \& Masdeu, J. C. (2003). Human Cerebral Activation during Steady-State Visual-Evoked Responses. Journal of Neuroscience, 23(37).

Picton, T. W., John, M. S., Dimitrijevic, A., \& Purcell, D. (2003). Human auditory steady-state responses: Respuestas auditivas de estado estable en humanos. International Journal of Audiology, 42(4), 177-219. https://doi.org/10.3109/14992020309101316

Radin, D. (2017). Electrocortical correlations between pairs of isolated people: A reanalysis. F1000Research, 6, 676. https://doi.org/10.12688/f1000research.11537.1

Stober, S., Sternin, A., Owen, A. M., \& Grahn, J. A. (2016). Deep Feature Learning for EEG Recordings. arXiv.

Walach, H., Tressoldi, P., \& Pederzoli, L. (2016). Mental, behavioural and physiological nonlocal correlations within the Generalized Quantum Theory framework. Axiomathes, 26(3), 313-328. https://doi.org/10.1007/s10516-016-9290-6 


\section{Supplemental Information}

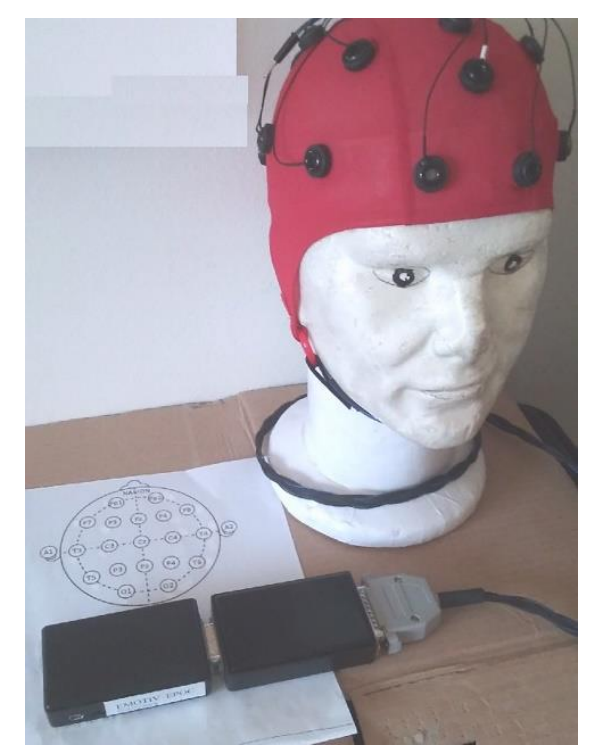

Figure S1: EEG headset and modified Emotiv Epoc ${ }^{\mathrm{TM}}$

\section{Pilot investigation using 15 and $18 \mathrm{~Hz}$ frequencies}

This is a summary of the preliminary research in preparation for the study representing the aim of this work. This preliminary research allowed us to better choose the frequencies to use in the definitive study and to improve experimental conditions, so much so that the final data produced proved to be of excellent quality.

The same experimental method as that described under Materials and Methods was used, but somewhat simplified, so that a higher number of tests could be performed in less time: in particular, only the EEG activity of the non-stimulated subjects were recorded. Furthermore, the stimuli (about 100 for each stimulated partner) were provided with the Steady-State modality at the modulation frequencies of $15 \mathrm{~Hz}$ and $18 \mathrm{~Hz}$.

The sensory and electrical separation of the two subjects was good, but sometimes external noises could still reach them. Because the applied stimuli were numerous and on-off modulated, we believe these noises did not pose any significant influence on final results.

Data were collected from 20 pairs of subjects who all got along well (mostly friends) and the results are described in Figures S2 and S3, which only show graphs from the GW6 method, in that values relative to power and normal ERPs were shown to be insignificant (as in graphs from Figure 7). 


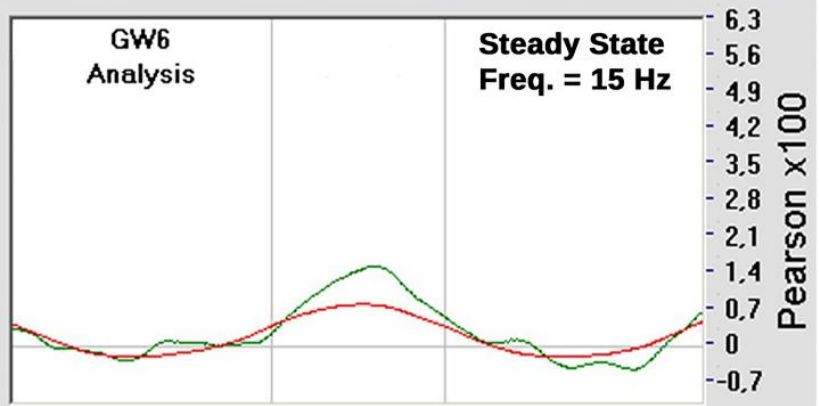

Time (4 seconds)

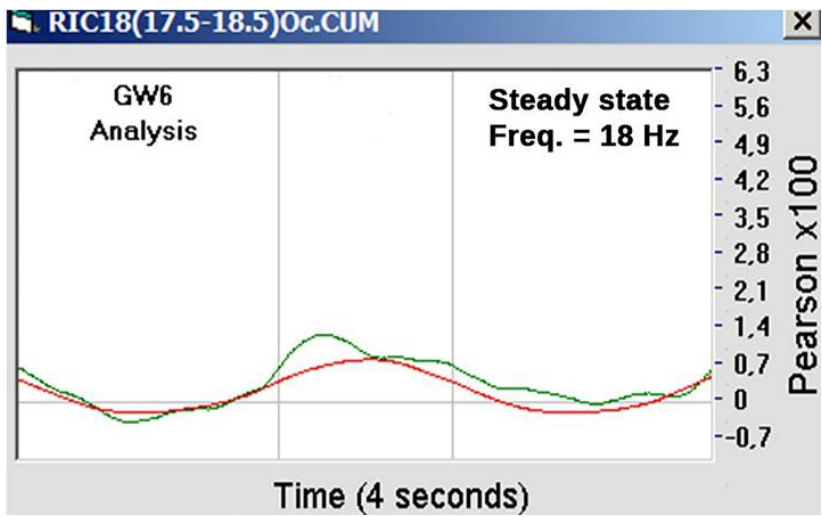

Time (4 seconds)

Figure S2: On the left, the average of 100 stimuli for 20 files filtered in the $14-15 \mathrm{~Hz}$ band. On the right the average of 100 stimuli for 20 files filtered in the $17-18 \mathrm{~Hz}$ band.

Graphs from Figures S2 and S3 show a large response (green curve) compared to what is expected by chance (red curve). The corresponding average values are presented in the Table S1.

Table S1 - Probability of the experimental max correlation compared to the random correlation.

\begin{tabular}{cccccc}
\hline $\begin{array}{c}\text { Stimulus } \\
\text { Frequency }\end{array}$ & $\begin{array}{c}\text { Experimental } \\
\text { max correlation } \\
\text { Mean (SD) }\end{array}$ & $\begin{array}{c}\text { Random } \\
\text { max correlation } \\
\text { Mean (SD) }\end{array}$ & $\begin{array}{c}\text { paired } \\
\text { t-test }\end{array}$ & $\begin{array}{c}p \text { value } \\
\text { (one-tailed) }\end{array}$ & $\begin{array}{c}p \text { value (one-tailed) } \\
\text { following the } \\
\text { Montecarlo procedure }\end{array}$ \\
\hline $\mathbf{1 5 ~ H z}$ & $1.24(1.18)$ & $0.72(1.11)$ & 2.09 & 0.03 & 0.06 \\
$\mathbf{1 8 ~ H z}$ & $1.18(0.91)$ & $0.79(0.94)$ & 1.81 & 0.06 & 0.1 \\
\hline
\end{tabular}

